\title{
Stiffness-Force Feedback in UAV Tele-operation
}

\author{
T. M. Lam, M. Mulder and M. M. van Paassen \\ Delft University of Technology \\ The Netherlands
}

\section{Introduction}

In the tele-operation of an uninhabited aerial vehicle (UAV), the operator and vehicle are physically separated. The limited sources of information of the environment, e.g., lack of a complete outside view, sounds, vehicle motions and vibrations, often lead to poor situation awareness. Tele-operation usually involves the use of a visual interface on a ground station, providing a navigation display and an outside visual generated by a camera mounted onboard the vehicle. The visual information, however, is usually not sufficient due to for instance a limited field of view, leading to an often not very efficient and sometimes even unsafe tele-operation (Elhaij et al., 2001; McCarley \& Wickens, 2005).

Previous research efforts indicated that a haptic interface, using force feedback via a haptic control device, can be used to complement the visual interface. Haptic feedback provides information about the environment through the sense of touch (Lam et al., 2004; Lam et al., 2007). Indeed, the multi-sensory haptic interface improved operator performance and significantly reduced the number of collisions, leading to an overall highly increased level of safety (Lam et al., 2007). Operator control activity and workload, however, increased as well. This may be attributed to the incompatibility between the haptic interface and the operator's intentions, based on her or his internal representation of the environment.

High operator control activity and workload particularly occur in situations where the UAV is surrounded by many obstacles. Although force feedback would indeed help the operator to avoid a collision with one obstacle, through deflecting the control device away from the direction of the obstacle, it may also direct the UAV towards another obstacle located at the other side. Whereas the haptic interface does not know about the other closely-located obstacle, the operator might already have located it from the visual interface, and would prefer to adjust the direction of motion only "just enough" to avoid collision.

The reported incompatibilities between the motions of the haptic device and the operator's intentions may have been caused by the particular implementation of the haptic interface, i.e., through applying force feedback alone. Here, an external force offset will actively deflect the haptic control device, in such a way that the vehicle moves away from the direction of an obstacle. The offset force, a function of the relative position and velocity of the UAV with respect to the obstacle, still exists even after the operator releases her hand from the control device and may cause the control device to deflect to the other side of the zero deflection. For control of a UAV helicopter, the focus of our study, the control deflection represents a velocity command. Therefore, the force feedback sometimes does not allow the operator to "rest" and follow the motions of the control device, but forces the 
operator to counteract them to avoid collision with another obstacle. This all contributes to high operator control activity and workload.

This chapter introduces two alternative implementations of haptic feedback: stiffness feedback and stiffness-force feedback. These alternatives aim to relieve the operator from continuously counteracting the repulsive forces from the haptic control device. The study involves both a theoretical discussion on the differences between the haptic feedback alternatives, as well as a pilot-in-the-loop experimental evaluation.

\section{Haptic feedback}

The use of haptic feedback through a control device allows the operator to perceive tactile cues through the sense of touch. The tactile information can represent various physical or mechanical properties, such as temperature level and relative distance (Elhaij et al., 2001). For collision avoidance in the tele-operation of a moving vehicle, it would be compatible with the operator's internal representation when the tactile cues would represent "repulsive forces" exerted from obstacles located near-by in the environment. These "repulsive forces" can be generated by an artificial force field (AFF) (Boschloo et al., 2004; Krogh, 1984).

The purpose of the artificial force field is to transform the UAV position and velocity relative to an obstacle, to a certain "value" that can be fed back to a haptic control device, yielding an impedance on the operator's control deflections. Fig. 1 shows a schematic representation of haptic feedback from a haptic control device, in our study a side-stick, to the human operator.

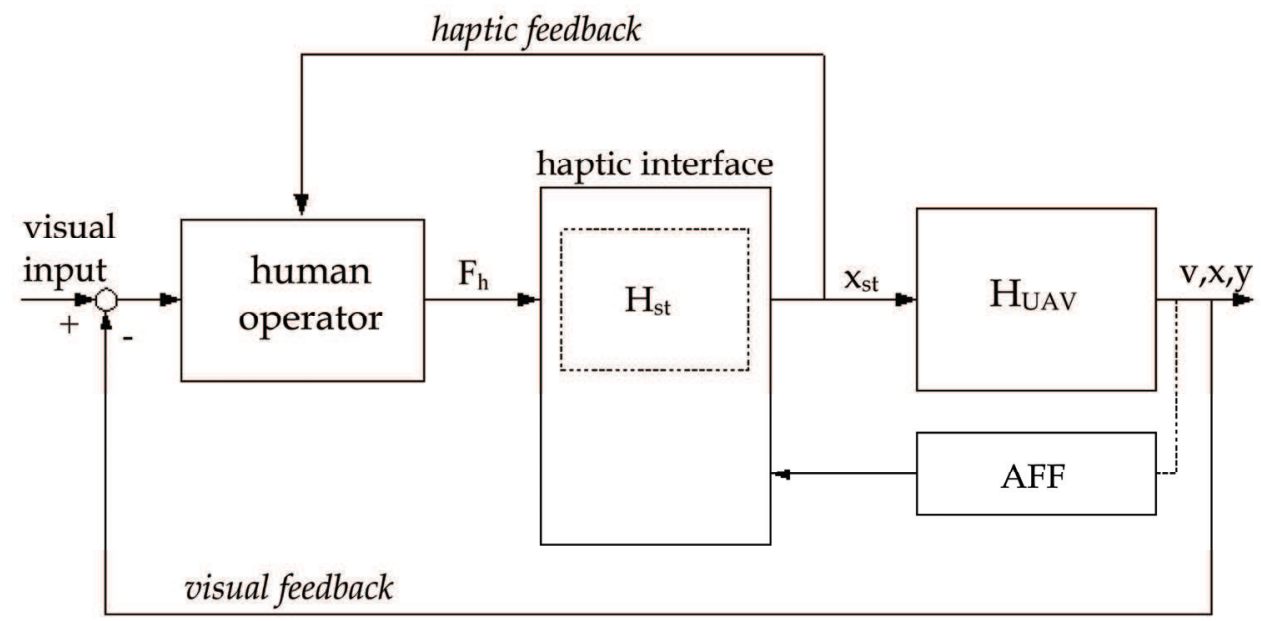

Figure 1. Schematic representation of a human-in-the-loop system with visual and haptic feedback

The impedance of the haptic control device consists of the stick mass-spring-damper dynamics $\mathrm{H}_{\text {st }}$, depicted in Eq. (1), and the extra impedance from the output of the AFF.

$$
\mathrm{H}_{\mathrm{st}}(\mathrm{s})=\frac{1}{\mathrm{~ms}^{2}+\mathrm{bs}+\mathrm{k}},
$$


with $\mathrm{m}, \mathrm{b}$ and $\mathrm{k}$ the stick's mass, damping coefficient and spring constant, respectively. The generated value of the AFF can be fed back in many different ways to the haptic device. In the following sections, three alternatives will be discussed.

\subsection{Force feedback}

In force feedback, a force offset $F_{f}$ is applied to the control manipulator to guide the operator, see Fig. 2. The total force that the operator perceives is the sum of the reaction force from the stick dynamics, $F_{s t}$, and the external force offset. Assume that the stick is displaced to a certain position, $\mathrm{x}_{\mathrm{st}}$, then the force exerted by the operator, i.e., the force on the hand, $F_{h}$, is written as:

$$
\begin{gathered}
\mathrm{F}_{\mathrm{h}}\left(\mathrm{x}_{\mathrm{st},} \mathrm{i}\right)=\mathrm{F}_{\mathrm{st}}\left(\mathrm{x}_{\mathrm{st}}\right)+\mathrm{F}_{\mathrm{f}}(\mathrm{i}), \\
\mathrm{F}_{\mathrm{h}}\left(\mathrm{x}_{\mathrm{st}} \mathrm{i}\right)=\mathrm{kx} \mathrm{x}_{\mathrm{st}}+\mathrm{F}_{\mathrm{f}}(\mathrm{i}),
\end{gathered}
$$

with $F_{s t}\left(x_{s t}\right)$ and $F_{f}(i)$ the reaction force from the control device as function of the stick deflection $\mathrm{x}_{\mathrm{st}}$, and the external force offset as function of haptic feedback information $\mathrm{i}$, respectively. Note that, with haptic feedback, the stick deflection $\mathrm{x}_{\mathrm{st}}$ acts as the input to the system to be controlled, in our case the UAV helicopter.

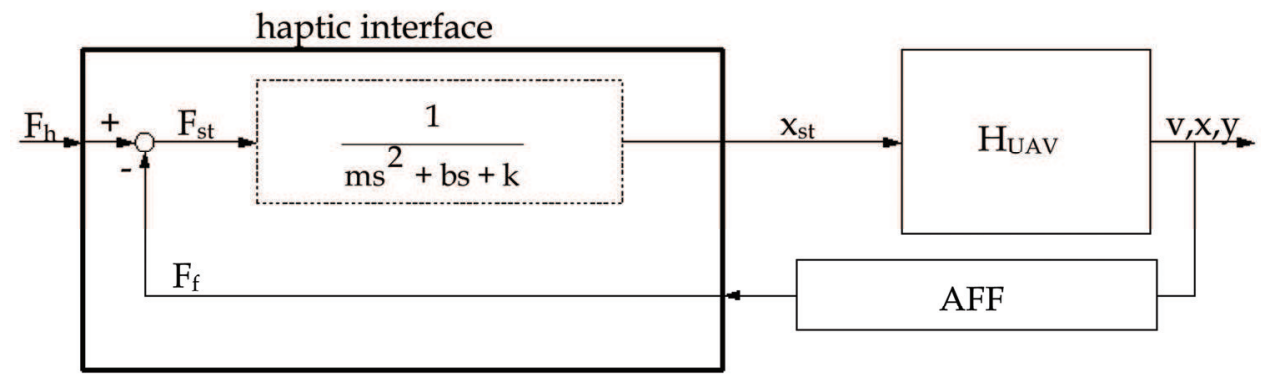

Figure 2. Schematic representation of the force feedback algorithm

Fig. 3 shows that due to the force offset, the stick will have a non-zero neutral position (A), i.e., the position where the stick is in equilibrium in the absence of external forces. When the stick is released, $F_{h}\left(x_{s t}, i\right)=0$, repulsive forces can still exist and the stick indeed actively deflects away from the direction with a possible collision: $\mathrm{x}_{\mathrm{st}}=-\mathrm{F}_{\mathrm{f}}(\mathrm{i}) / \mathrm{k}$. This active deflection with hands-off can be considered as an "autonomous collision avoidance" function. In fact, the force feedback can be regarded to yield a "commanded" stick deflection that the operator should follow as good as possible. That is, when yielding to the forces applied on the hand, the operator would deflect the stick in a way that satisfies the collision avoidance function. Because a control device is generally limited in its deflections, there is also a limit to the amplitude of the force feedback, i.e., a natural constraint to the force feedback gain. In previous studies, Lam et al. found that the amplitudes of stick motions due to neutral position changes may contribute to workload (Lam et al., 2007; Lam et al., 2008). In particular when flying through a narrow corridor, or when moving along multiple smaller and closely-spaced obstacles, these force offsets may vary continuously. The operator is not able to follow the force feedback accurately and overshoots and control oscillations occur. 


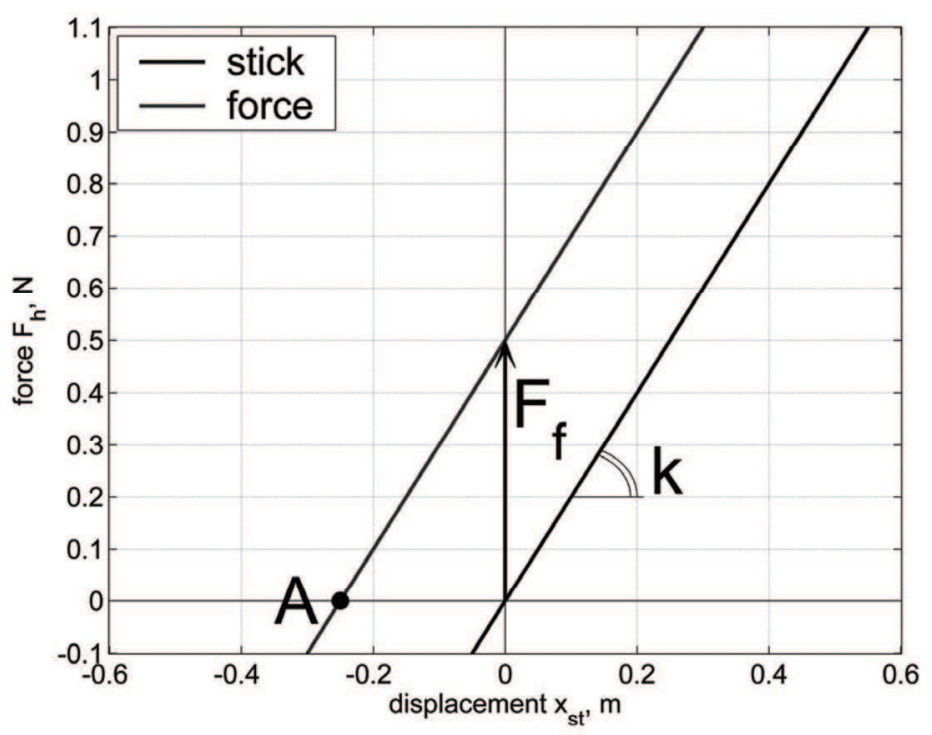

Figure 3. Force-displacement relation of a force feedback system

A possible solution would be to reduce the force offset magnitude, i.e., to decrease the gain of the force feedback. This might result, however, in repulsive forces that are too small in magnitude, in particular for operators who adopt a high neuromuscular stiffness. It would therefore significantly reduce the effectiveness of the collision avoidance command and limits the haptic presentation of information regarding a potential collision. Tuning the force feedback magnitude needs a compromise between, on the one hand, the effectiveness of the haptic interface and, on the other hand, the workload it imposes on the operator.

\subsection{Stiffness feedback}

Stiffness feedback involves addition of an extra spring load, $\mathrm{k}_{\mathrm{s}}(\mathrm{i})$ as a function of haptic feedback information i, to the nominal stick dynamics' spring constant k, see Fig. 4. Instead of having a force offset, the stick becomes stiffer when in the presence of an obstacle, that is, the extra stiffness provides an impedance, resulting in an extra force that depends on the deflection of the stick by the operator. When the stick is released it will not actively deflect away from a possible collision, as is the case when using force feedback, but just returns to the neutral position. Hence, stiffness feedback alone is not suitable for implementing autonomous collision avoidance. The total force that an operator perceives in this situation can be written as:

$$
\begin{gathered}
F_{h}\left(x_{s t}, i\right)=F_{s t}\left(x_{s t}\right)+F_{s}\left(x_{s t}, i\right), \\
F_{h}\left(x_{s t}, i\right)=k x_{s t}+k_{s}(i) x_{s t .}
\end{gathered}
$$

with $\mathrm{F}_{\mathrm{s}}\left(\mathrm{x}_{\mathrm{st}}, \mathrm{i}\right)$ the force due to an extra spring load $\mathrm{k}_{\mathrm{s}}(\mathrm{i})$. Fig. 5 shows that the slope of the force-excursion relation increases due to the extra spring load. The line rotates around the origin, and therefore a zero displacement leads to zero repulsive force. 


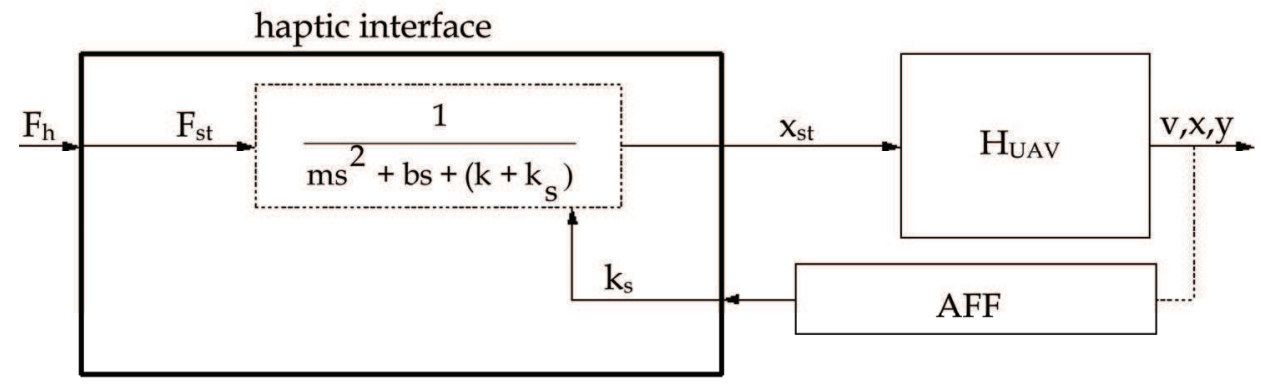

Figure 4. Schematic representation of the stiffness feedback algorithm

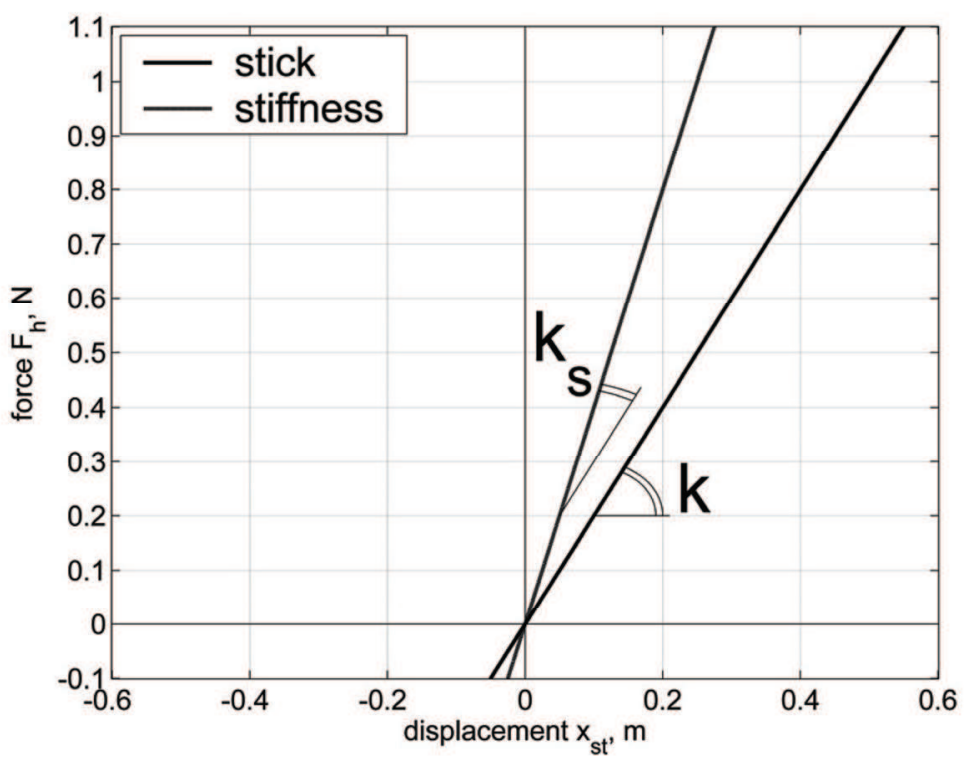

Figure 5. Force-displacement relation of a stiffness feedback system

Since small displacements result in relatively small repulsive forces, human operators may not perceive sufficient information when using only small stick displacements. With a zero stick displacement the operator will not be provided with any haptic feedback. When in this case the UAV would have moved very close to the obstacle, like in the case of drift due to wind, the stiffness feedback would have become so large that the stick cannot be moved at all. Providing only stiffness feedback is therefore undesirable. The haptic device should be capable of actively deflecting the stick away from a possible collision, preferably with only small overshoot from the zero displacement in order to not introduce control problems. It could be helpful, however, when the haptic device would be able to provide large resistance when the stick is deflected in the "wrong" direction, i.e., with high risk of collision. Hence, when combining the force feedback with stiffness feedback these two properties of haptic feedback can be achieved. This combination will be discussed below. 


\subsection{Stiffness-force feedback}

The lack of active repulsive deflections of the control device with stiffness feedback can be resolved by combining it with force feedback, see Fig. 6 . In this combination, referred to as "stiffness-force" feedback, the total exerted force by the human operator is defined as:

$$
\begin{gathered}
F_{h}\left(x_{s t}, i\right)=F_{s t}\left(x_{s t}\right)+F_{s}\left(x_{s t}, i\right)+F_{f}(i), \\
F_{h}\left(x_{s t}, i\right)=k x_{s t}+k_{s}(i) x_{s t}+F_{f}(i) .
\end{gathered}
$$

Fig. 7 shows the force-excursion relation of stiffness-force feedback. A property of this configuration is that due to the increase in stiffness, the desired offset of the neutral position, (A), commanded by the force feedback will decrease, which results in (B). It can also be seen, however, that in the hands-off case $\left(\mathrm{F}_{\mathrm{h}}\left(\mathrm{x}_{\mathrm{st}}, \mathrm{i}\right)=0\right)$ the stick deflection $\mathrm{x}_{\mathrm{st}}$ becomes equal to $\mathrm{F}_{\mathrm{f}}(\mathrm{i}) /\left(\mathrm{k}+\mathrm{k}_{\mathrm{s}}(\mathrm{i})\right)$, i.e., the stiffness feedback actually reduces the effects of the force feedback.

\section{Haptic interface}

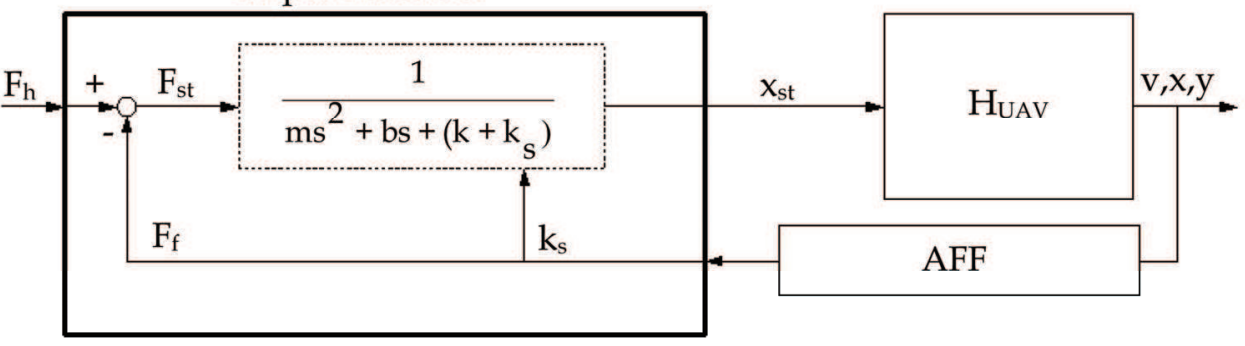

Figure 6. Schematic representation of the stiffness-force feedback algorithm

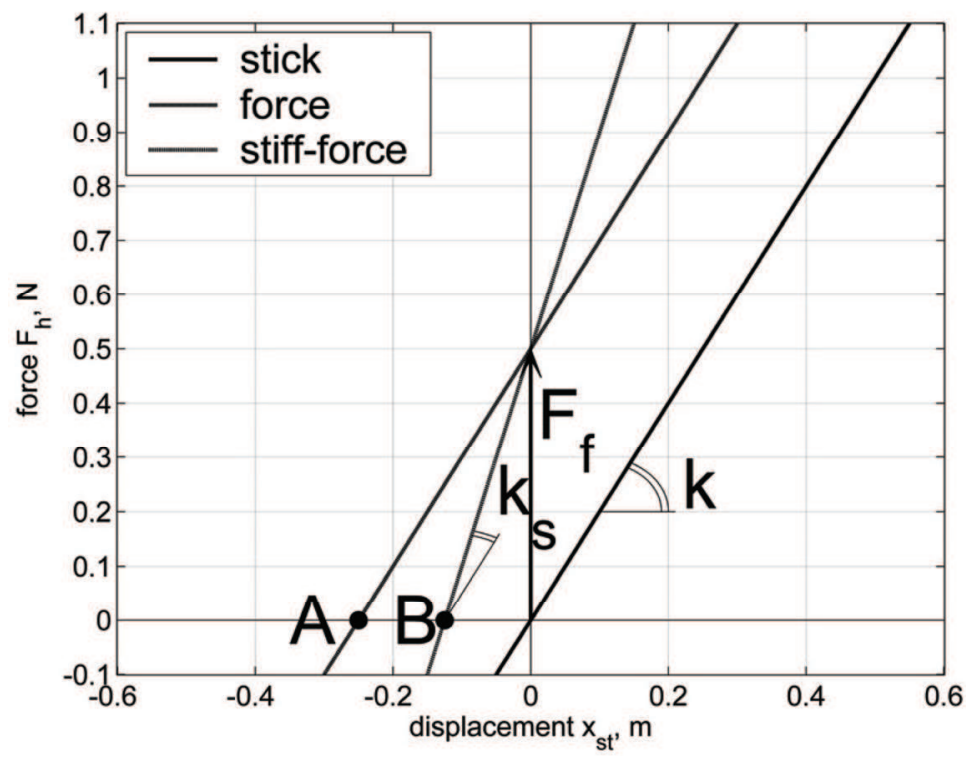

Figure 7. Force-displacement relation of a stiffness-force feedback system 


\subsection{Stability analysis}

As mentioned in the introduction, force feedback may cause the stick to overshoot the neutral position. Operators sometimes need to counteract the active motions, particularly when obstacles are present at the other side. This indicates that active stick motion, a property of force feedback, could be a source for undesired oscillations. This section will consider the stability of force feedback, stiffness feedback and stiffness-force feedback at the hand of computer simulations, using the closed-loop system from Fig. 1. The human operator model contained a neuromuscular model for the lateral direction from (Lam et al., 2005). The stick dynamics were described as:

$$
H_{s t}(s)=\frac{1}{0.015 s^{2}+0.3 s+3} .
$$

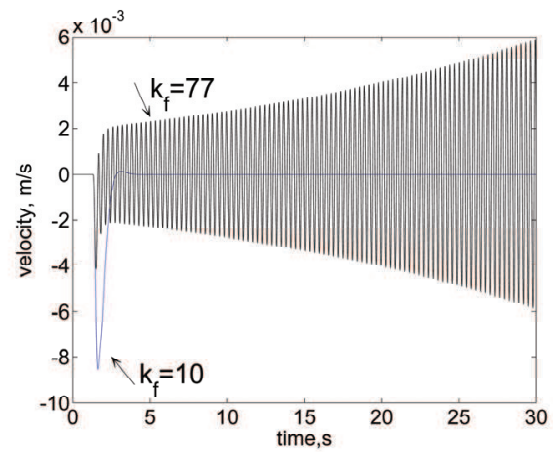

a) force feedback

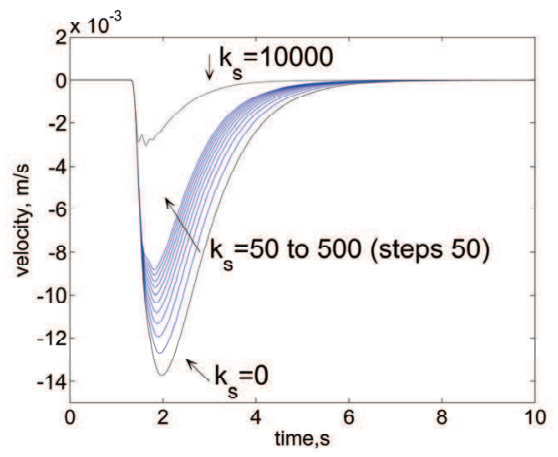

b) stiffness feedback

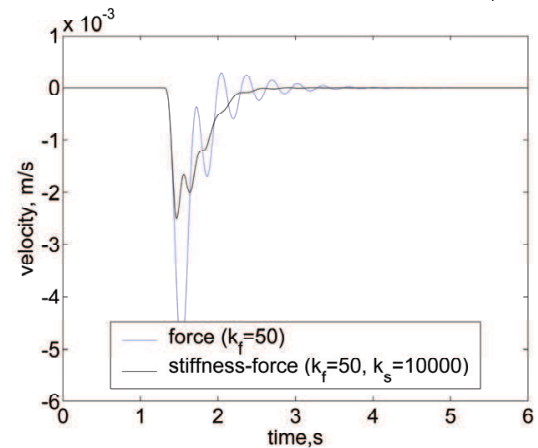

c) stiffness-force feedback

Figure 8. Step responses of the different haptic feedback configurations

The UAV helicopter was assumed to be control-augmented, with dynamics equivalent to a first order relation between stick deflection and UAV velocity (Voorsluijs et al., 2004):

$$
\mathrm{H}_{\mathrm{UAV}}(\mathrm{s})=\frac{2}{\mathrm{~s}+2} \text {. }
$$


The function of the AFF was to generate a value, based on relative position and velocity toward an obstacle, which could be used as input to the haptic device. Since this theoretical investigation only focuses on the behavior of the closed-loop system response to the different feedback of the AFF signal, the actual (non-linear) algorithm of AFF was left out and replaced by a gain for direct feedback of the velocity. For force feedback and stiffness feedback, the gains were $\mathrm{k}_{\mathrm{f}}$ and $\mathrm{k}_{\mathrm{s}}$, respectively. It was assumed that the AFF primarily depended on the velocity.

Figs. 8(a) and 8(b) show step responses of a force feedback and stiffness feedback for increasing $\mathrm{k}_{\mathrm{f}}$ and $\mathrm{k}_{\mathrm{s}}$ gains, respectively. Fig. 8(c) shows the influence of stiffness feedback on a step response with stiffness-force feedback. It can be seen that a step response with force feedback becomes unstable for large force gain $k_{f} \geq 77$, whereas the stiffness feedback will not result in instability. For large stiffness gains $k_{s}$ the stick dynamics will become "infinitely" stiff and no deflection will occur at all. The better stability and no change of neutral position suggests that stiffness feedback would result in less workload and control activity. Additionally, stiffness feedback has a reducing effect on force feedback, as was already illustrated in Fig. 7.

\section{Tuning of the stiffness feedback}

Both force feedback and stiffness feedback will cause the human operator to exert a larger force to keep the same deflection. However, the two configurations have a different contribution to the repulsive force. The force feedback is directly mapped as a repulsive force, whereas the stiffness feedback is only an increase of the spring constant. Hence, when a same value from the AFF is used to generate external force or external spring constant, the latter will result in a smaller repulsive force, which also depends on the stick deflection.

Because the side stick in the experiment uses moments and angles instead of forces and displacements, we will refer to moments and angles from this point. When considering a hand that is exerting a certain moment $\mathrm{M}_{\mathrm{h}}$ on the haptic device the following holds:

$$
\begin{aligned}
& M_{h}\left(\theta_{s t}, i\right)=M_{s t}\left(\theta_{s t}\right) \quad+M_{\text {ext }}\left(\theta_{s t}, i\right), \\
& M_{h}\left(\theta_{s t}, i\right)=\underbrace{\ddot{I \theta}_{s t}+B \dot{\theta}_{s t}+K \theta_{s t}}_{\text {stick dynamics }}+\underbrace{M_{f}(i)+M_{s}\left(\theta_{s t}, i\right)}_{\begin{array}{c}
\text { haptic } \\
\text { dynamics }
\end{array}},
\end{aligned}
$$

with I, B, and $\mathrm{K}$ the stick moment of inertia, damping coefficient, and spring constant, respectively. $\theta_{\mathrm{st}}$ is the stick rotation, $\mathrm{M}_{\mathrm{st}}\left(\theta_{\mathrm{st}}\right)$ is the reaction moment from the stick dynamics, and $\mathrm{M}_{\text {ext }}(\mathrm{i})$ is the repulsive moment from the haptic device. $\mathrm{M}_{\text {ext }}(\mathrm{i})$ may consist of a moment offset $\mathrm{M}_{\mathrm{f}}(\mathrm{i})$ from force feedback and/or $\mathrm{M}_{\mathrm{s}}(\mathrm{i})$ from the stiffness feedback, due to extra spring constant.

To compare force feedback and stiffness feedback, it is important to tune the stiffness feedback in such a way that both systems will generate approximately the same level of repulsive force. The strategy adopted in this chapter is to tune the maximum repulsive moment based on the so-called Parametric Risk Field (PRF), an AFF adopted from (Boschloo et al., 2004). 
For a maximum risk value of 1 (the limit of the Parametric Risk Field) and a force gain $\mathrm{k}_{\mathrm{f}}=1.5$, the maximum repulsive moment $\left(\mathrm{M}_{\mathrm{ext}}\left(\theta_{\mathrm{st}}, \mathrm{i}\right)\right)$ the force feedback can generate is 1.5 $\mathrm{Nm}$. For tuning the stiffness gain, the maximum repulsive moment provided by stiffness feedback should also be $1.5 \mathrm{Nm}$. Consider the contribution of stiffness to the haptic dynamics:

$$
M_{s}\left(\theta_{s t}, i\right)=K_{s}(i) \theta_{s t} .
$$

The maximum repulsive moment from stiffness feedback depends on the extra spring constant $K_{s}(\mathrm{i})$ and the maximum stick deflection, which was $\theta_{\text {st(long) }}=0.35$ rad and $\theta_{\text {st(lat) }}=$ $0.4 \mathrm{rad}$ in the longitudinal and lateral direction, respectively. Hence, for $\mathrm{M}_{\mathrm{ext}}\left(\theta_{\mathrm{st}}, \mathrm{i}\right)=$ $\mathrm{M}_{\mathrm{s}}\left(\theta_{\mathrm{st}}, \mathrm{i}\right)=1.5 \mathrm{Nm}$ the stiffness gain for the longitudinal and lateral direction is:

$$
\begin{aligned}
\mathrm{K}_{\mathrm{s}(\text { long })} & =\frac{1.5}{0.35}=4.29 \mathrm{Nm} / \mathrm{rad}, \\
\mathrm{K}_{\mathrm{S}(\text { lat })} & =\frac{1.5}{0.40}=3.75 \mathrm{Nm} / \mathrm{rad} .
\end{aligned}
$$

Pure stiffness feedback, however, has some drawbacks as mentioned in Section 2.2. First, the repulsive force generated by the stiffness feedback only equals the force feedback when the stick is maximally deflected. Second, for small deflections, small repulsive forces are generated that are very hard to sense. Third, in case of drifting toward an obstacle due to wind the operator would not feel any feedback (assuming there is no controller, rejecting the drift), because the stick is not deflected into the direction of the obstacle. But the stiffness would also be very high, making it difficult for the operator to deflect the stick away from the direction of a potential collision.

It is, therefore, recommended to use stiffness-force feedback with a small part of force feedback in order to provide some feedback when small or no deflections are given, while the UAV is in a close vicinity of an obstacle. Through trial and error, it was decided to use $20 \%$ and $40 \%$ of the force offset in the longitudinal and lateral direction, respectively. Now, for a maximal deflection, the total external moment can exceed $1.5 \mathrm{Nm}$ due to the partial addition of the force feedback. Since operators are able to release the stick, resulting in a rapid decrease of the repulsive force to avoid large overshoot, it is unlikely that the higher maximum external moment would lead to larger workload and control activity.

\section{Experiment}

A human-in-the-loop experiment was conducted to investigate the effects of the various force feedback and stiffness-force feedback alternatives on human operator performance, collision avoidance, control activity and workload.

\subsection{Subjects and instructions}

Eight subjects with no flight experience participated in the experiment. Their main task was to fly from waypoint to waypoint as accurately as possible in an obstacle-laden 
environment. The experiment simulated a reconnaissance task in a hazardous environment. The waypoints were represented by smoke plumes, strategically positioned near particular obstacles in the environment. Note that subjects were not provided with information whether or not, or when a collision occurred. After each run, a subject was asked to rate the workload she or he experienced using the NASA TLX rating scale (Hart \& Staveland, 1988).

\subsection{Apparatus}

The experiment was conducted in a fixed-base simulator. Subjects were seated on an aircraft chair in front of an 18 inch screen, presenting a navigation display (Fig. 9(a)), and looking forward towards a large white wall, on which the outside view from a hypothetical onboard camera was projected (Fig. 9(b)).

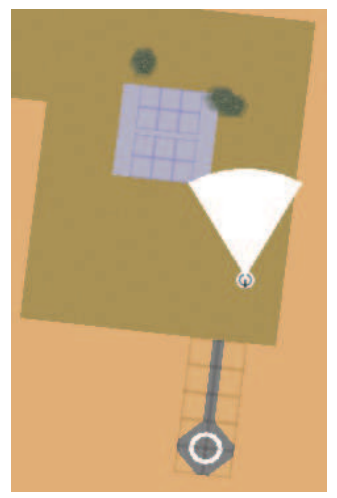

a) 2-dimensional navigation display

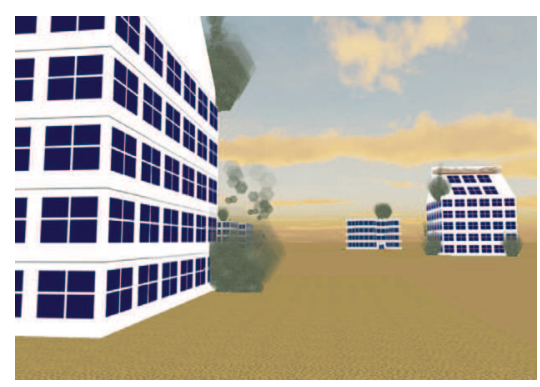

b) 3-dimensional camera display

Figure 9. Experimental navigation and camera displays

On the right side of the aircraft chair, an electro-hydraulic side stick was used as the haptic control device. The mass-spring-damper stick dynamics were simulated with the inertia I = $0.01 \mathrm{kgm}^{2}$, damping coefficient $\mathrm{B}=0.2 \mathrm{Nms} / \mathrm{rad}$ and spring constant $\mathrm{K}=2 \mathrm{Nm} / \mathrm{rad}$. The UAV was simulated by a control-augmented helicopter model, Eq. (9), with a maximum velocity of $5 \mathrm{~m} / \mathrm{s}$ and maximum acceleration of $1 \mathrm{~m} / \mathrm{s}^{2}$. The altitude was kept constant by the control augmentation.

\subsection{Independent variables}

Two independent variables were used in the experiment: three levels of haptic feedback (HF) and six levels of subtask (ST).

The three haptic feedback conditions were:

Haptic off (HFoff):

Force (HF1):

Stiffness-force (HF2):
Subjects would only feel the side stick simulated mass-springdamper dynamics;

Subjects would feel external forces due to force offset generated by the PRF (Boschloo et al., 2004); and

Subjects would feel external forces, due to external spring constant and partial force offset, also based on the PRF.

The six subtasks are listed below, with the item number corresponding to the subtask number. The subtasks are illustrated in Fig. 10, with an arrow showing the flight direction and the red 
stars indicating the waypoint location, i.e., the smoke plumes. Note that a secondary purpose of the smoke plumes was to reduce the visibility of the obstacle boundaries.

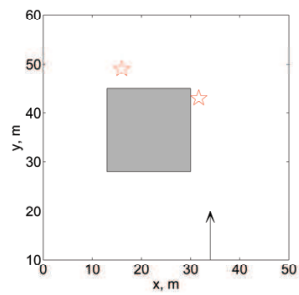

a) subtask 1

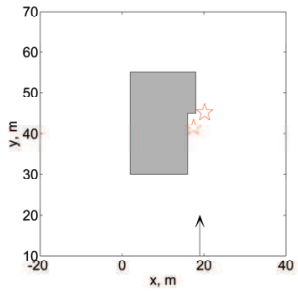

d) subtask 4

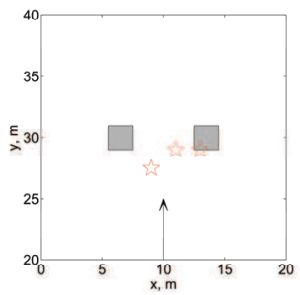

b) subtask 2

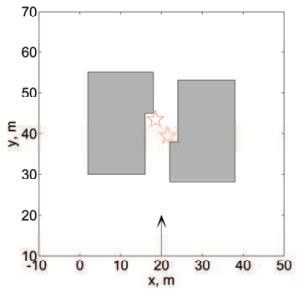

e) subtask 5

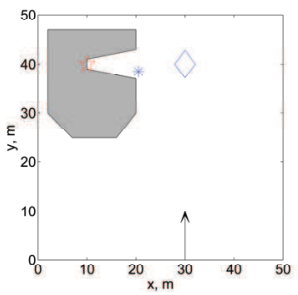

c) subtask 3

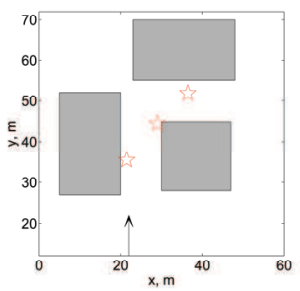

f) subtask 6

Figure 10. Top-down view of the six experimental subtasks

Subtask 1 In this subtask, the helicopter had to make a 90 degrees turn around a building, Fig. 10(a). Before the turn, the UAV had to approach the first waypoint, forcing the UAV to fly closely to the corner. The second waypoint was located after the corner in order to force subjects to make a turn as sharp as possible.

Subtask 2 In this subtask, the helicopter was to fly through a narrow gate of which the posts served as two closely-spaced, small obstacles. Fig. 10(b) shows a cross section of the gate. The smoke plume positioned between the posts was meant to reduce the visibility of the posts, making it more difficult to fly through the gate, rather than that it should serve as a waypoint.

Subtask 3 This subtask demanded a special task during hover. Once the helicopter had reached the (blue) diamond, it should hover backward toward the building until the operator could see a certain stop sign that was below the flight altitude and fixed in the world (blue asterisk), Fig. 10(c). Here, the camera would not point in the direction of motion and it was expected that haptic feedback would become very useful. Smoke on top of the building was placed to reduce the visibility of the edges of the building, leading to difficult estimation of the building edges, rather than to serve as waypoint.

Subtask 4 This subtask consisted of a building with a discrete change in the shape of the wall. The smoke was located before the discrete change of the wall and forced the UAV to approach the wall followed by an escape maneuver to avoid collision with the extension of the wall, Fig. 10(d). The second smoke plume was placed in order to force the UAV to stay close to the building during the escape maneuver. 
Subtask 5 In this subtask, two buildings with discrete changes in opposite directions might lead to oscillatory behaviour in the stick and might cause considerable control difficulties. The first location of the smoke plume would force the UAV to make a sharp turn, whereas the second smoke plume would force the UAV to make an escape maneuver, Fig. 10(e).

Subtask 6 In this subtask, the turn radius with haptic feedback would be limited due to the obstacles in front and at the left side. It was expected that this subtask would lead to control difficulties, when approaching with high speed. The first smoke plume would force the UAV to approach the side of a building, whereas the second smoke plume would force the UAV to make a quick turn to fly closely along the corner of the building. The third smoke plume would force the UAV to approach another wall after the turn, Fig. 10(f).

\subsection{Trajectory}

In general, the trajectories (scenarios) were the same as was used in (Lam et al., 2007). The difference was the absence of a reference path in this experiment. For navigation, the subtask areas had a darker background colour on the navigation display (Fig. 9(a)).

The trajectories consisted of three sectors, which were in turn clusters of the six subtasks in a different order. To prevent boredom, 6 different trajectories were designed. Each trajectory contained a different order of the sectors. Each trajectory was flown once for each condition. Hence, each subject had to fly $6 \times 3=18$ runs; each run took approximately 5 min.

\subsection{Dependent measure}

The performance of collision avoidance was expressed by the number of collisions (cnt). The level of safety was expressed by the minimum allowable distance toward an obstacle $\left(D_{\min }\right)$. The performance of tele-operation was expressed by the minimum distance from the smoke location $\left(\mathrm{D}_{\text {smoke }}\right)$. Speed-related measures were expressed by the average of the total speed $(\bar{v})$ and the standard deviation of the total speed $\left(\sigma_{\mathrm{v}}\right)$.

The standard deviations of the total stick deflection $\sigma_{\delta \text { tot }}$ and the total exerted moment by the hand $\left(\sigma_{\mathrm{Mh}}\right)$ represented control activity. Haptic activity was represented by the standard deviation of the total external moment by the haptic device $\left(\sigma_{\text {Mext }}\right)$. Workload was measured using the NASA TLX rating scale, after each measurement run.

\subsection{Procedure}

Each subject flew 6 runs for each haptic configuration. Before the actual experiment, subjects got the opportunity to get familiar with the three haptic configurations by training runs. After each experiment run, subjects were asked to rate their workload using the NASA TLX rating scale. After the experiment, subjects were asked to complete a questionnaire, regarding their experience with the three haptic feedback configurations.

\subsection{Results and discussion}

The main results are discussed in this section. A full-factorial ANOVA was applied. The means and 95\% confidence intervals of the dependent measures are shown in Fig. 12. 


\subsubsection{Collisions}

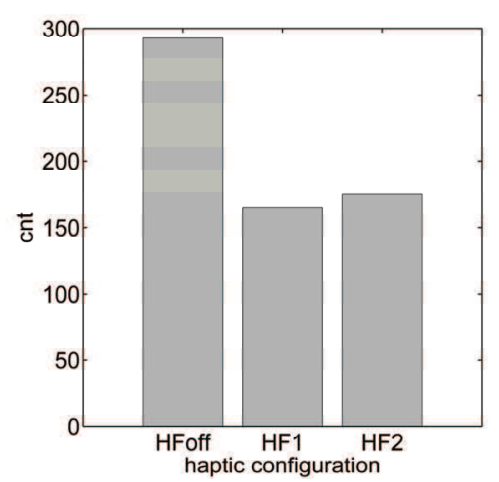

Figure 11. Total number of collisions (data of all subjects)

The total number of collisions, illustrated in Fig. 11, was highest when flying without haptic feedback, which was expected. The absence of haptic feedback, HFoff, led to a lack of situation awareness, especially when the visual information was reduced due to the smoke plumes. The force feedback, HF1, resulted in slightly less collisions than the stiffness-force feedback, HF2.

\subsubsection{Approach performance}

Performance expressed in terms of the distance from the smoke plumes (the waypoints) is shown in Fig. 12(a). Subtasks 2 and 3 were not included, because here the smoke plumes were placed with the objective to reduce the visual information of the obstacle boundaries, rather than to serve as a waypoint. Fig. 12(a) shows that HF1 resulted in the largest distance from the waypoints, whereas no haptic feedback resulted in the smallest distance from the waypoints, a highly-significant effect $\left(\mathrm{HF}: \mathrm{F}_{2,14}=8.129, \mathrm{p} \leq 0.01\right)$. A post-hoc analysis revealed that the stiffness-force feedback lies in-between the force feedback and no haptic feedback conditions (Student-Newman Keuls (SNK), $\alpha=0.05$ ). Regarding the effect of subtask, in subtasks 1 and 4 , subjects were able to fly closely to the smoke. In subtasks 5 and 6 , with objects surrounding the vehicle, subjects were not able to closely approach the waypoints, due to the difficult escape maneuver the close approach would cause afterwards, a highly-significant effect (ST: $\left.\mathrm{F}_{3,21}=10.024, \mathrm{p} \leq 0.01\right)$.

\subsubsection{Control activity}

Fig. 12(b) indicates no significant effect of HF on the stick deflections. In subtask 3, subjects had to fly backward and forward, resulting in the largest standard deviation of the stick deflection. In subtask 2, subjects only had to fly straight through a passage, resulting in the smallest standard deviation of the stick deflection, a highly-significant effect (ST: $F_{5,35}=$ 29.343, p $\leq$ 0.01). Fig. 12(c) shows that without haptic feedback the standard deviation of the measured moment was significantly smallest (HF: $\left.\mathrm{F}_{2,14}=8.173, \mathrm{p} \leq 0.01\right)$. Post-hoc analysis (SNK, $\alpha=0.05$ ) revealed that the difference between force and stiffness-force feedback was not significant. Similar to the stick deflection, subtask 3 and subtask 2 resulted in the largest and smallest variation of the measured moment, respectively (ST: $\left.F_{5,35}=33.592, p \leq 0.01\right)$. 


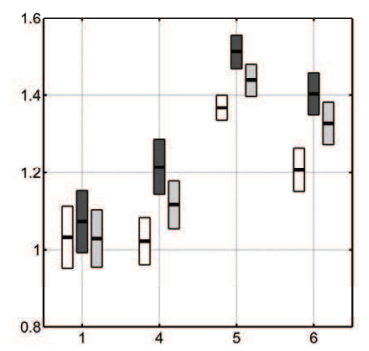

a) $D_{\text {smoke, }} m$

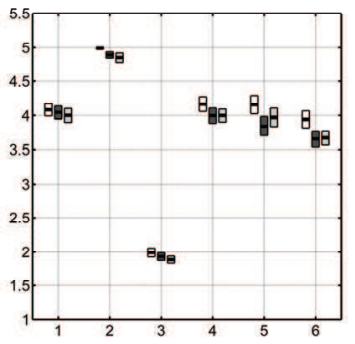

d) $\bar{v}, \mathrm{~m} / \mathrm{s}$

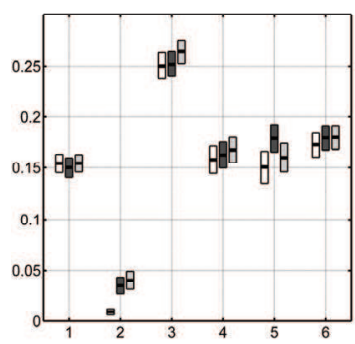

b) $\sigma_{\delta \text { tot }}, \mathrm{rad}$

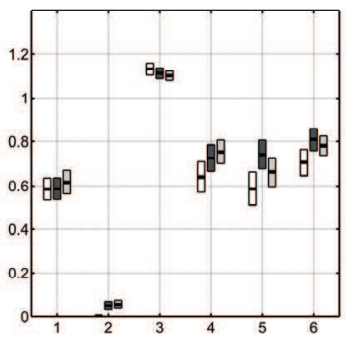

e) $\sigma_{v}, \mathrm{~m} / \mathrm{s}$

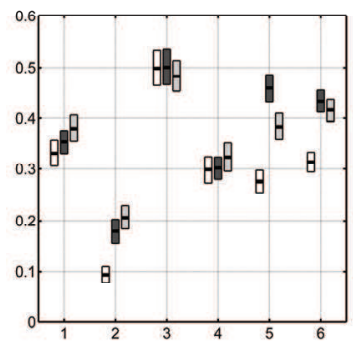

c) $\sigma_{\mathrm{Mh}}, \mathrm{Nm}$

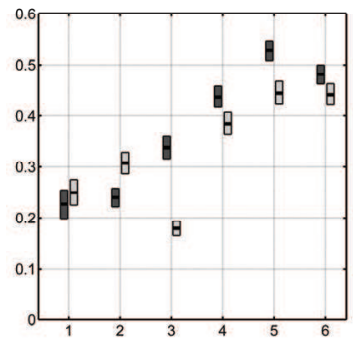

f) $\sigma_{\text {Mext }}, \mathrm{Nm}$

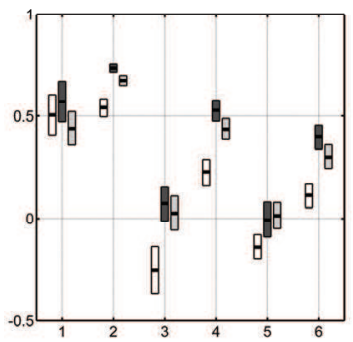

g) $\mathrm{D}_{\min }, \mathrm{m}$

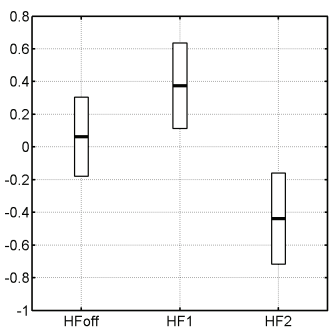

h) $\mathrm{TLX}_{\mathrm{z}}$ -

Figure 12. The means and 95\% confidence intervals of the main dependent measures. The numbers 1 to 6 on the horizontal axis correspond to the subtask numbers. In all subfigures except (h), the white, dark gray, and light gray bars represent the haptic feedback conditions HFoff, HF1, and HF2, respectively

\subsubsection{Haptic activity}

Fig. 12(f) indicates that the standard deviation of the external moment was highlysignificantly larger for the force feedback condition (HF: $\left.F_{1,7}=39.700, p \leq 0.01\right)$. Obviously, the condition without haptic feedback was not considered. Subtasks 4, 5 and 6, all demanding a difficult approach and escape maneuver, resulted in large variations of the external moment $\left(\mathrm{ST}: \mathrm{F}_{5,35}=28.255, \mathrm{p} \leq 0.01\right)$. In subtask 2 , the external moment was larger for the stiffness-force feedback; this may be due to the partial addition of force feedback as discussed in the theoretical analysis section. 


\subsubsection{Speed}

The average of the UAV velocity, a measure of tele-operation efficiency, was highlysignificantly larger without haptic feedback, due to the lack of the repulsive forces in the opposite flight direction (HF: $F_{2,14}=7.338, p \leq 0.01$ ), Fig. 12(d). Post-hoc analysis revealed that the difference between HF1 and HF2 was not significant (SNK, $\alpha=0.05)$. In subtask 2 (short and relatively easy) and 3 (difficult), the average speed was highly-significantly largest and smallest, respectively (ST: $\left.F_{5,35}=59.978, p \leq 0.01\right)$. Fig. 12(e) shows that subtasks 2 and 3 resulted in a highly-significantly smallest and largest standard deviation of the total speed, respectively $\left(S T: F_{5,35}=24.635, \mathrm{p} \leq 0.01\right)$.

\subsubsection{Minimum distance}

Fig. 12(g) shows that HFoff resulted in the smallest distance, whereas HF1 resulted in the largest distance, a highly-significant effect $\left(\mathrm{HF}: \mathrm{F}_{2,14}=24.209, \mathrm{p} \leq 0.01\right)$. Subtasks 3 and 5 resulted in the smallest distance from a building, a highly-significant effect (ST: $\mathrm{F}_{5,35}=$ 19.058, $\mathrm{p} \leq$ 0.01). Note that a negative distance was due to collisions and the smallest distance due to HFoff corresponded to the largest amount of collisions as discussed in Section 4.7.1.

\subsubsection{Workload}

Fig. 12(h) shows the TLX z-scores and indicates that stiffness-force feedback, as expected, resulted in the lowest workload, a significant effect $\left(\mathrm{HF}: \mathrm{F}_{2,14}=5.888, \mathrm{p}=0.014\right)$.

\section{Conclusions and recommendations}

It can be concluded that, when considering the approach performance and workload, stiffness-force feedback outperforms force feedback with equivalent control activity, level of safety and UAV velocities. The improved approach performance and lower workload can be attributed to the relatively lower repulsive force activity with stiffness-force feedback.

The additional stiffness changes the dynamics of the haptic interface, which influences the interactions with the operator. A stiffer side stick would make it more difficult for the operator to overrule the force feedback. Hence, stiffness feedback in combination with force feedback will not only add extra repulsive force, but it may also serve as a haptic feedback design parameter to establish the level of operator autonomy. In order to achieve this, however, the reducing effect of stiffness feedback on force feedback should be eliminated. The first steps towards this new concept, provisionally called "force-stiffness feedback" have proven to be successful (Abbink \& Mulder, 2008).

\section{References}

Abbink, D. A. \& Mulder, M. (2008), Exploring the Dimensions of Haptic Feedback Support in Manual Control, Journal of Computing and Information Science Engineering, accepted

Boschloo, H. W.; Lam, T. M.; Mulder, M. \& Van Paassen, M. M. (2004), Collision Avoidance for a Remotely-Operated Helicopter Using Haptic Feedback, Proceedings of the IEEE International Conference on Systems, Man and Cybernetics, The Hague, The Netherlands, 10-13 October, pp. 229-235 
Elhaij, I.; Xi, N.; Fung, W. K.; Liu, Y. H.; Li, W. J.; Kaga, T. \& Fukuda, T., (2001). Haptic Information in Internet-Based Tele-operation, IEEE/ASME Transactions on Mechatronics, Vol. 6, No. 3, pp. 295-304

Hart, S. G. \& Staveland, L. E. (1988), Development of NASA-TLX (Task Load Index): Results of Empirical and Theoretical Research, Hancock, Human Mental Workload, P. A. and Meshkati, N., pp. 139-183, Elsevier Science Publishers (North-Holland)

Krogh, B. H. (1984), A Generalized Potential Field Approach to Obstacle Avoidance Control, Society of Manufacturing Engineers Technical Paper, No. MS84-484.

Lam, T. M.; Boschloo, H. W.; Mulder, M.; Van Paassen, M. M. \& Van der Helm, F. C. T. (2004), Effect of Haptic Feedback in a Trajectory Following Task with an Unmanned Aerial Vehicle, Proceedings of IEEE International Conference on Systems, Man and Cybernetics, The Hague, The Netherlands, 10-13 October, pp. 2500-2506

Lam, T. M.; Mulder, M.; Van Paassen, M. M. \& Van der Helm, F. C. T. (2005), Tele-operating a UAV Using Haptics - Modeling the Neuromuscular System, Proceedings of the IEEE International Conference on Systems, Man and Cybernetics, Hawaii, USA, 10-12 October, pp. 2695-2700

Lam, T. M.; Mulder, M. \& Van Paassen, M. M. (2007), Haptic Interface for UAV Collision Avoidance, International Journal of Aviation Psychology, Vol. 17, No. 2, pp. 167-195

Lam, T. M.; Mulder, M. \& Van Paassen, M. M. (2008), Haptic Feedback in UAV Teleoperation with Time Delay, AIAA Journal of Guidance, Control, and Dynamics, Vol. 31, No. 6, pp. 1728-1739

McCarley, J. S. \& Wickens, C. D. (2005), Human Factors Implications of UAVs in the National Airspace, Technical report Aviation Human Factors Division, AHFD-0505/FAA-05-01, pp. 1-5, Savoy, Illinois

Voorsluijs, G. M.; Bennani, S. \& Scherer, C. W. (2004), Linear and Parameter-dependent Robust Control Techniques Applied to a Helicopter UAV, Proceedings of the 38-th AIAA Guidance, Navigation and Control Conference, Providence (RI), August 16-19, AIAA paper 2004-4909. 


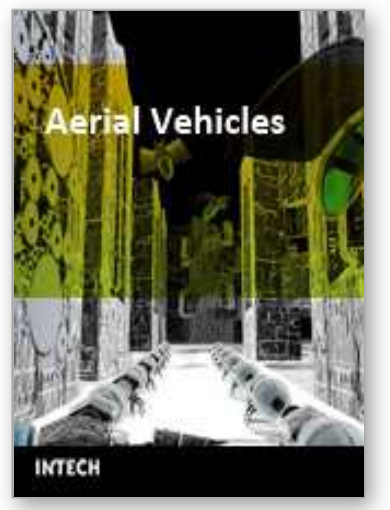

\author{
Aerial Vehicles \\ Edited by Thanh Mung Lam
}

ISBN 978-953-7619-41-1

Hard cover, 320 pages

Publisher InTech

Published online 01, January, 2009

Published in print edition January, 2009

This book contains 35 chapters written by experts in developing techniques for making aerial vehicles more intelligent, more reliable, more flexible in use, and safer in operation.It will also serve as an inspiration for further improvement of the design and application of aeral vehicles. The advanced techniques and research described here may also be applicable to other high-tech areas such as robotics, avionics, vetronics, and space.

\title{
How to reference
}

In order to correctly reference this scholarly work, feel free to copy and paste the following:

T. M. Lam, M. Mulder and M. M. van Paassen (2009). Stiffness-Force Feedback in UAV Tele-Operation, Aerial Vehicles, Thanh Mung Lam (Ed.), ISBN: 978-953-7619-41-1, InTech, Available from: http://www.intechopen.com/books/aerial_vehicles/stiffness-force_feedback_in_uav_tele-operation

\section{INTECH}

open science | open minds

\author{
InTech Europe \\ University Campus STeP Ri \\ Slavka Krautzeka 83/A \\ 51000 Rijeka, Croatia \\ Phone: +385 (51) 770447 \\ Fax: +385 (51) 686166 \\ www.intechopen.com
}

\author{
InTech China \\ Unit 405, Office Block, Hotel Equatorial Shanghai \\ No.65, Yan An Road (West), Shanghai, 200040, China \\ 中国上海市延安西路65号上海国际贵都大饭店办公楼405单元 \\ Phone: +86-21-62489820 \\ Fax: $+86-21-62489821$
}


(C) 2009 The Author(s). Licensee IntechOpen. This chapter is distributed under the terms of the Creative Commons Attribution-NonCommercialShareAlike-3.0 License, which permits use, distribution and reproduction for non-commercial purposes, provided the original is properly cited and derivative works building on this content are distributed under the same license. 